\title{
High-Energy Carrier-Envelope Phase-Stable Optical Waveforms Compressible to $<1$ fs Using Induced-Phase Modulation in an Argon-Filled Hollow-Core Fiber
}

\author{
Shaobo Fang, ${ }^{1,3, *}$ Hong Ye,${ }^{1,2}$ Giovanni Cirmi, ${ }^{1,3}$ Shih-Hsuan Chia, ${ }^{1,2}$ Sergio Carbajo,,${ }^{1,2}$ \\ Oliver D. Mücke, ${ }^{1,3}$ and Franz X. Kärtner ${ }^{1-4}$ \\ ${ }^{1}$ Center for Free-Electron Laser Science, Deutsches Elektronen-Synchrotron DESY, \\ Notkestraße 85, 22607 Hamburg, Germany \\ ${ }^{2}$ Physics Department, University of Hamburg, Luruper Chaussee 149, 22761 Hamburg, Germany \\ ${ }^{3}$ The Hamburg Center for Ultrafast Imaging, Luruper Chaussee 149, 22761 Hamburg, Germany \\ ${ }^{4}$ Department of Electrical Engineering and Computer Science and Research Laboratory of Electronics, \\ Massachusetts Institute of Technology, Cambridge, Massachusetts 02139, USA \\ shaobo.fang@desy.de
}

\begin{abstract}
We demonstrate phase-stable, $\sim 0.4-\mathrm{mJ}$ optical waveforms based on induced-phase modulation for generating sub-fs optical pulses. Using custom-designed double-chirped mirrors and a spatial light modulator, such optical waveforms will become a versatile tool for strong-field attoscience.
\end{abstract}

Ultrashort optical pulses with high energy and multi-octave bandwidth have been greatly desired for the generation of intense monocycle optical pulses. This is because those pulses are expected to be crucially important as driver pulses for efficient generation of isolated attosecond pulses with sub-100-as duration in the extreme-ultraviolet (XUV) region. However, the generation of such pulses with both high energy and multioctave bandwidth has been a very challenging subject so far. To overcome the problem, one of the most popular techniques is coherent optical waveform synthesis, which means the generation of custom-tailored, intense, fewcycle or even sub-cycle optical waveforms by coherently stitching together separate spectral portions. Currently, coherent waveform synthesis is one of the most intriguing and promising frontiers of attosecond science and strong-field physics, opening up unprecedented prospects, e.g., for precision control of strong-field interactions in atoms, molecules and solids, generation of intense isolated attosecond pulses, and attosecond pump-probe spectroscopy employing ultrashort pulses in the VIS/IR and XUV/soft-X-ray regions.

Up to now, coherent waveform synthesis based on self-phase modulation (SPM) in a neon-filled hollow-core fiber (HCF) compressor allowed for the generation of sub-cycle $\sim 300-\mu \mathrm{J}$ optical pulses [1]. For pursuing the synthesis of the shortest possible pulses within this scheme, the total output energy of this multi-channel synthesizer is limited to a few tens of $\mu \mathrm{Js}$ mainly by the UV channel containing the smallest pulse energy [2], thus preventing further energy upscaling to the (multi-)mJ level, which is required for many interesting applications in attosecond science. A potential solution out of this dilemma is the application of induced-phase modulation (IPM) [3] based on the interaction between two (or more) co-propagating optical pulses of different colors and relatively long pulse durations in a gas-filled HCF. The IPM technique offers control over the spectral shape by adjusting the relative intensity ratio and the relative delay between the input pulses and allows a more efficient generation of broader-band optical pulses than those produced solely by SPM. Such an IPM-based synthesizer is expected to greatly relieve the energy-scaling bottleneck in the UV region [4], and the enhanced spectral broadening of the UV region is particularly appealing for the realization of ultrahigh HHG conversion efficiencies in bright coherent tabletop high-harmonic sources.

In our earlier work, we already generated transform-limited (TL) 2.6-fs, 3.6- $\mu \mathrm{J}$ optical pulses centered at 600 $\mathrm{nm}$ by compensating for the chirp of the ultrabroad spectrum generated by IPM in an argon-filled HCF [3]. Later, by employing the pressure-gradient technique [5], the output energy was further scaled up to $860 \mu \mathrm{J}$, supporting 1.5 -fs transform-limited pulses. However, to investigate phenomena sensitive to the electric field rather than merely to the intensity of the laser pulse, such as high-harmonic generation and above-threshold ionization, the carrier-envelope phases (CEPs) of the different few-cycle pulses used in the synthesis must be locked. In this work, we demonstrate for the first time an IPM waveform synthesizer driven by a CEP-stabilized chirped-pulse amplification (CPA) system. By optimizing the energy ratio between the two input pulses, we generated a 380$\mu \mathrm{J}$ (a typical energy for an HHG driver pulse) CEP-locked supercontinuum spanning the range of 300-930 nm with nearly constant intensity, ideally suited for high-energy sub-femtosecond optical waveform generation. 


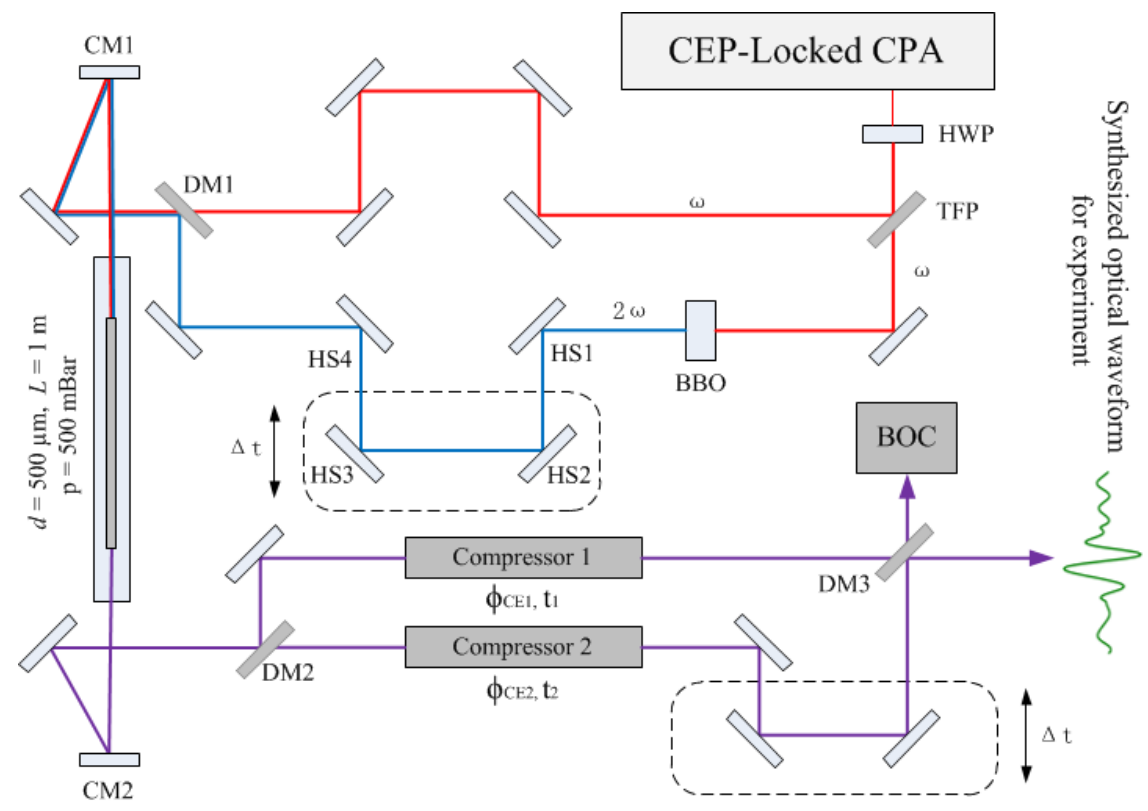

Fig. 1 Sub-femtosecond IPM-based optical waveform synthesizer driven by a CEP-stable CPA: DM, dichroic mirror ; HWP, half-wave plate; TFP, thin-film polarizer; HS, harmonic separator; CM, concave mirror; BOC, balanced optical cross-correlator.

In the experimental setup shown in Fig. 1, we started from a commercial CEP-stabilized Ti:sapphire CPA system $(790 \mathrm{~nm}, 3 \mathrm{kHz}, 30 \mathrm{fs}, 5.5 \mathrm{~mJ})$. The output was split into two beams with different polarizations using a thin-film polarizer (TFP) with ratio controlled by a half-wave plate (HWP). The reflected s-polarized pulses were used as fundamental pulse. The transmitted $p$-polarized pulses were frequency-doubled in a 0.5 -mm-thick Type-I $\beta$-barium borate (BBO) crystal to generate the second-harmonic $(2 \omega)$ pulses with the same $s$ polarization. Two pairs of harmonic separators (HSs) were used to filter out the residual fundamental pulses from the secondharmonic pulses, as well as to adjust the time delay $\Delta t$. The $\omega$ and $2 \omega$ pulses were recombined via a dichroic mirror and reflected by a concave silver mirror with a radius of curvature of 4 meters, which focused the combined beam into a fused-silica HCF (length: $L=1 \mathrm{~m}$, diameter: $d=500 \mu \mathrm{m}$ ). The fiber was placed in the middle of a 4-m-long glass tube sealed at the two ends with 3-mm-thick $\mathrm{CaF}_{2}$ windows at Brewster angle and filled with argon gas. In the current setup, the output supercontinuum from the HCF is measured using a spectrometer. To avoid any damage from the input end of the HCF, we first limited our total output energy from the laser system to $1 \mathrm{~mJ}$. After optimizing the energy ratio between the two input pulses, a fundamental pulse with $800-\mu \mathrm{J}$ energy and a second-harmonic pulse with $60-\mu \mathrm{J}$ energy (second-harmonic efficiency $30 \%$ ) were focused and injected into the HCF. We optimized the gas pressure (500 mbar) to achieve the broadest IPM output spectrum. Finally, we obtained $380 \mu \mathrm{J}$ from the HCF with spectrum spanning from $300 \mathrm{~nm}$ to $930 \mathrm{~nm}$, corresponding to 0.9-fs TL pulse duration (Fig. 2). After splitting this broadband light source into two different wavelength channels via a broadband dichroic mirror (DM), we plan to compress the different spectral regions using custom-designed double-chirped mirrors (DCM $\mathrm{Vis-NIR}_{\text {) }}$ and a UV spatial-light modulator (SLM) [6]. Finally, we will recombine the two channels by another broadband dichroic mirror. We also need to tightly lock the relative timing of the two pulses using feedback loop with a balanced optical cross-correlator (BOC), that can achieve sub-cycle synchronization with $<30$-as (rms) timing jitter $[7,8]$.

In conclusion, we have discussed the technological challenges of a novel IPM-based waveform synthesizer and presented the first experimental results. By employing pressure-gradient technique, custom-designed DCMs and a UV-SLM in future experiments, we foresee that our mJ-level sub-fs synthesizer will become a versatile tool for nonlinear attosecond optics experiments [9]. 


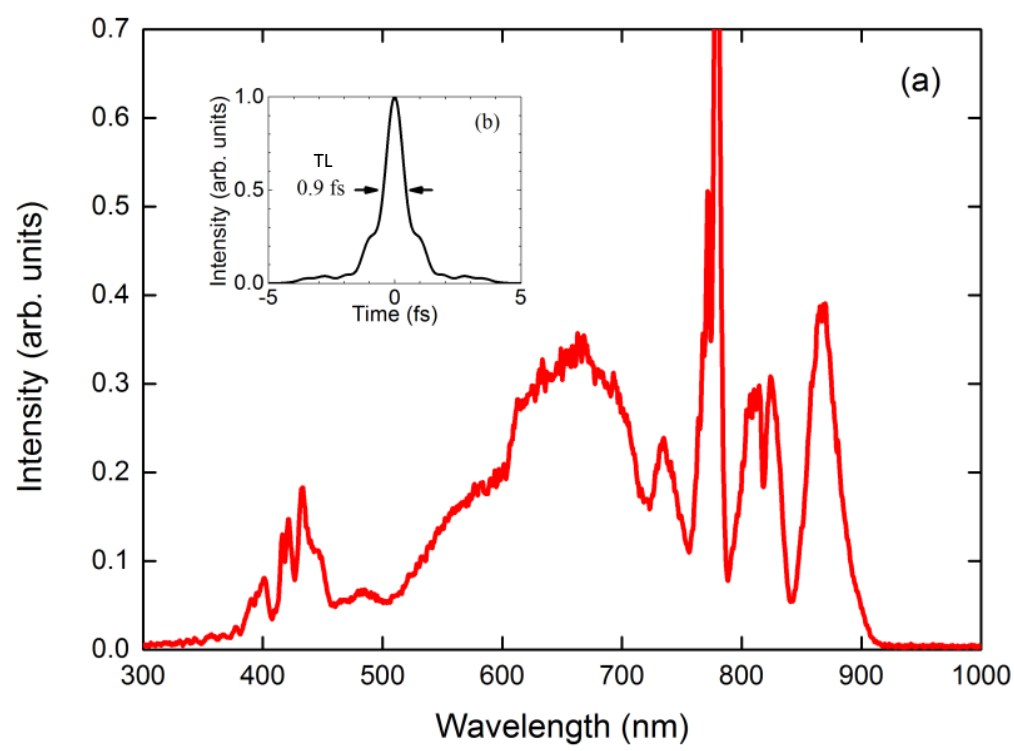

Fig. 2 Supercontinuum created by IPM (a), and the corresponding TL pulse (b).

[1] A. Wirth et al., "Synthesized Light Transients," Science 334, 195-200 (2011).

[2] T. T. Luu et al., QELS 2013, paper QF1C.6 (2013).

[3] E. Matsubara et al., "Generation of 2.6 fs optical pulses using induced-phase modulation in a gas filled hollow fiber," JOSA B 24, 985989 (2007)

[4] S. Fang et al., "Multi-mJ Parametric Synthesizer Generating Two-Octave-Wide Optical Waveforms," invited talk WB3-1, CLEO Pacific Rim, Kyoto, Japan, (2013).

[5] S. Fang et al., "Generation of Sub-900- $\mu \mathrm{J}$ Supercontinuum With a Two-Octave Bandwidth Based on Induced Phase Modulation in Argon-Filled Hollow Fiber,” IEEE Phot. Tech. Lett. 23, 688-690 (2011).

[6] T. Tanigawa et al.,"Spatial light modulator of 648 pixels with liquid crystal transparent from ultraviolet to near-infrared and its chirp compensation application," Opt. Lett. 34, 1696-1698 (2009).

[7] S.-W. Huang et al., "High-energy pulse synthesis with sub-cycle waveform control for strong-field physics," Nature Photonics 5, 475 479 (2011)

[8] C. Manzoni et al., "Coherent synthesis of ultra-broadband optical parametric amplifiers," Opt. Lett. 37, 1880-1882 (2012).

[9] E. J. Takahashi et al., "Attosecond nonlinear optics using gigawatt-scale isolated attosecond pulses,” Nat. Commun. 4:2691, (2013). 